\title{
EXPLICIT M/G/1 WAITING-TIME DISTRIBUTIONS FOR A CLASS OF LONG-TAIL SERVICE-TIME DISTRIBUTIONS
}

\author{
by \\ Joseph Abate ${ }^{1} \quad$ Ward Whitt ${ }^{2}$ \\ AT\&T retired AT\&T Labs
}

February 5, 1998

Operations Research Letters 25 (1999) 25-31

${ }^{1} 900$ Hammond Road, Ridgewood, NJ 07450-2908

${ }^{2}$ Room A117, AT\&T Labs, 180 Park Avenue, Building 103, Florham Park, NJ 07932-0971; email: wow@research.att.com 
O. J. Boxma and J. W. Cohen recently obtained an explicit expression for the $M / G / 1$ steady-state waiting-time distribution for a class of service-time distributions with power tails. We extend their explicit representation from a one-parameter family of service-time distributions to a two-parameter family. The complementary cumulative distribution function (ccdf's) of the service times all have the asymptotic form $F^{c}(t) \sim \alpha t^{-3 / 2}$ as $t \rightarrow \infty$, so that the associated waiting-time ccdf's have asymptotic form $W^{c}(t) \sim \beta t^{-1 / 2}$ as $t \rightarrow \infty$. Thus the second moment of the service time and the mean of the waiting time are infinite. Our result here also extends our own earlier explicit expression for the $M / G / 1$ steady-state waiting-time distribution when the service-time distribution is an exponential mixture of inverse Gaussian distributions (EMIG). The EMIG distributions form a two-parameter family with ccdf having the asymptotic form $F^{c}(t) \sim \alpha t^{-3 / 2} e^{-\eta t}$ as $t \rightarrow \infty$. We now show that a variant of our previous argument applies when the service-time ccdf is an undamped EMIG, i.e., with ccdf $G^{c}(t)=e^{\eta t} F^{c}(t)$ for $F^{c}(t)$ above, which has the power tail $G^{c}(t) \sim \alpha t^{-3 / 2}$ as $t \rightarrow \infty$. The Boxma-Cohen long-tail service-time distribution is a special case of an undamped EMIG.

Keywords: M/G/1 queue, waiting-time distribution, Pollaczek-Khintchine formula, long-tail distributions, power-tail distributions, exponential mixture of inverse Gaussian distributions. 


\section{Introduction}

The steady-state waiting-time distribution in the $\mathrm{M} / \mathrm{G} / 1$ queue is available via the classical Pollaczek-Khintchine transform. It can be readily computed by numerical transform inversion, when the service-time Laplace transform is available, e.g., as shown in Abate and Whitt [1]. Nevertheless it is interesting to have explicit formulas. When the service-time distribution has a rational transform, so does the waiting-time distribution, and the transform can be inverted analytically. More generally, the transform can be inverted analytically, yielding the Beneš formula, which is an infinite series containing $n$-fold convolutions of the service-time stationary-excess distribution for all $n$; e.g., see 4.82 on p. 255 of Cohen [8]. Because of the complexity of the Beneš formula, however, it is natural to look for more explicit formulas.

A more explicit formula for a non-rational service-time distribution was evidently first obtained for the gamma service-time distribution with shape parameter $1 / 2$ in (9.21) of Abate and Whitt [1]. This result was extended in Proposition 8.2 of Abate and Whitt [3] to all exponential mixtures of inverse Gaussian (EMIG) service-time distributions. These servicetime distributions have probability densities with asymptotics of the form $f(t) \sim \alpha t^{-3 / 2} e^{-\eta t}$ as $t \rightarrow \infty$, where $f(t) \sim g(t)$ as $t \rightarrow \infty$ means that $f(t) / g(t) \rightarrow 1$. Because of the $e^{-\eta t}$ term, these EMIG distributions do not have a long (a heavy) tail. However, recently, Boxma and Cohen [7] obtained an explicit expression for the $\mathrm{M} / \mathrm{G} / 1$ waiting-time distribution for a class of long-tail service-time distributions. In this paper, we extend Boxma and Cohen's result to a larger class of long-tail service-time distributions. In particular, we extend our result in [3] to undamped EMIGs, i.e., to distributions with complementary cumulative distribution functions (ccdf's) $G^{c}(t) \equiv 1-G(t)=e^{\eta t} F^{c}(t)$, where $F^{c}(t)$ is an EMIG ccdf. The Boxma-Cohen service-time distributions are a subclass.

Here is how the rest of this paper is organized. In Section 2 we give the explicit solution for the steady-state waiting-time distribution. In Section 3 we show that the service-time distributions used in Section 2 can be represented as undamped EMIGs. In Section 4 we show that both EMIGs and undamped EMIGs are completely monotone (mixtures of exponentials) and give their mixing densities. In Section 5 we give the asymptotic behavior of undamped EMIGs as $t \rightarrow 0$ and as $t \rightarrow \infty$. We apply that result to give the first two terms of the asymptotic expansion for the waiting-time ccdf in Section 2, which agrees with Boxma and Cohen [7]. In Section 6 we discuss the heavy-traffic approximation due to Boxma and Cohen [7]. For the service-time distributions considered here, we derive their limit from the explicit 
waiting-time ccdf. We conclude in Section 7 by discussing other service-time distributions for which explicit representations of the waiting-time distribution are possible, but the greater complexity make them of dubious value.

\section{The Explicit Solution}

Consider a service-time probability density function (pdf) $g(t)$ with Laplace transform

$$
\hat{g}(s) \equiv \int_{0}^{\infty} e^{-s t} g(t) d t=1-\frac{s}{(\mu+\sqrt{s})(1+\sqrt{s})},
$$

which has mean $m_{1}(g)=\mu^{-1}$ and all higher moments infinite. The pdf $g$ has two-parameters, the displayed $\mu$ and the scale, which has been omitted. Both can range over the positive reals.

The Pollaczek-Khintchine formula involves the associated stationary-excess pdf $g_{e}(t) \equiv$ $\mu G(t), t \geq 0$. Its Laplace transform has the nice form

$$
\hat{g}_{e}(s) \equiv \frac{1-g(s)}{s m_{1}(g)}=\frac{\mu}{(\mu+\sqrt{s})(1+\sqrt{s})} .
$$

For $\mu \neq 1$,

$$
\hat{g}_{e}(s)=\left(\frac{\mu}{1-\mu}\right)\left(\frac{1}{\mu+\sqrt{s}}-\frac{1}{1+\sqrt{s}}\right),
$$

so that, by 29.3.37 of Abramowitz and Stegun [6],

$$
g_{e}(t)=\mu G^{c}(t)=\left(\frac{\mu}{1-\mu}\right)\left(\psi(t)-\mu \psi\left(\mu^{2} t\right)\right), \quad t \geq 0
$$

where

$$
\psi(t) \equiv e^{t} \operatorname{erfc}(\sqrt{t}) \sim \frac{1}{\sqrt{\pi t}} \quad \text { as } \quad t \rightarrow \infty,
$$

with $\operatorname{erfc}$ being the complementary error function, i.e.,

$$
\operatorname{erf} c(t) \equiv \frac{2}{\sqrt{\pi}} \int_{t}^{\infty} e^{-u^{2}} d u \equiv 2 \Phi^{c}(\sqrt{2} t)
$$

where $\Phi^{c}(t) \equiv 1-\Phi(t)$ is the standard (mean 0, variance 1) normal complementary cumulative distribution function (ccdf); see 7.1.1 and 26.2.29 of Abramowitz and Stegun [6]. We will establish further properties of $G$ and $G_{e}$ in the next section.

The case $\mu=1$ was considered by Boxma and Cohen [7]. The case $\mu=1$ also corresponds to a subclass of beta mixtures of exponential (BME) pdf's considered by Abate and Whitt [4]; we will discuss this connection further in the next section. Boxma and Cohen show that the service-time ccdf when $\mu=1$ is

$$
G^{c}(t)=(2 t+1) \psi(t)-2 \sqrt{t / \pi}, \quad t \geq 0
$$


for $\psi$ in (2.5). In the next section we will show that the associated stationary-excess ccdf is

$$
G_{e}^{c}(t)=2 \sqrt{t / \pi}-(2 t-1) \psi(t), \quad t \geq 0
$$

We now consider the steady-state waiting-time distribution in the $\mathrm{M} / \mathrm{G} / 1$ queue with arrival rate $\lambda$. It has an atom of $1-\rho$ at the origin, assuming that $\rho \equiv \lambda / \mu<1$, but otherwise a pdf. The Laplace transform of the ccdf is

$$
\hat{W}^{c}(s)=\frac{\rho}{s}\left(1-\hat{w}_{\rho}(s)\right)
$$

where $\hat{w}_{\rho}(s)$ is the Laplace transform of the conditional waiting time pdf, given that there is a positive wait, i.e.,

$$
\hat{w}_{\rho}(s)=\frac{(1-\rho) \hat{g}_{e}(s)}{1-\rho \hat{g}_{e}(s)} .
$$

Paralleling Propoosition 8.2 of Abate and Whitt [3], we can find an explicit expression for $\hat{W}^{c}(s)$ and analytically invert it. From $(2.2)-(2.10)$, we deduce the following.

Theorem 2.1. For the service-time pdf $g(t)$ with Laplace transform $\hat{g}(s)$ in (2.1),

$$
\hat{w}_{\rho}(s)=\frac{(1-\rho) \mu}{\nu_{1}-\nu_{2}}\left(\frac{1}{\nu_{2}+\sqrt{s}}-\frac{1}{\nu_{1}+\sqrt{s}}\right)
$$

and

$$
\hat{W}^{c}(s)=\frac{\rho}{\nu_{1}-\nu_{2}}\left(\frac{\nu_{1}}{\sqrt{s}\left(\nu_{2}+\sqrt{s}\right)}-\frac{\nu_{2}}{\sqrt{s}\left(\nu_{1}+\sqrt{s}\right)}\right)
$$

so that

$$
W^{c}(t)=\frac{\rho}{\nu_{1}-\nu_{2}}\left(\nu_{1} \psi\left(\nu_{2}^{2} t\right)-\nu_{2} \psi\left(\nu_{1}^{2} t\right)\right),
$$

where $\psi$ is given in (2.5) and

$$
\nu_{1,2}=\frac{1+\mu}{2} \pm \sqrt{\left(\frac{1+\mu}{2}\right)^{2}-(1-\rho) \mu} .
$$

Proof. Algebra yields (2.11) and (2.12). The Laplace transform (2.12) is easy to invert using 29.3.43 of Abramowitz and Stegun [6].

The case $\mu=1$ (with $v_{1}=1+\sqrt{\rho}$ and $v_{2}=1-\sqrt{\rho}$ ) was obtained by Boxma and Cohen [7]. They included an atom at the origin in the service-time distribution, which we could do as well. The atom at the origin simply gets absorbed in $\rho$, i.e., corresponds to changing the arrival rate $\lambda$. This property is most easily seen from the virtual waiting time, which has the same distribution as the actual waiting time in $\mathrm{M} / \mathrm{G} / 1$. A customer with 0 service time causes no change in the virtual waiting-time process upon its arrival. By the Poisson thinning property, 
the arrival process of customers with positive service times is also a Poisson process but with reduced arrival rate $\lambda(1-\eta)$, where $\eta$ is the atom at 0 in the service-time distribution. Hence, having an atom of mass $\eta$ at 0 in the service-time distribution is equivalent to changing the arrival rate to $\lambda(1-\eta)$ and considering the service-time distribution without the atom, i.e., the conditional service-time distribution given that it is positive.

\section{Undamped EMIGs}

We obtain the service-time transform $\hat{g}(s)$ in (2.1) by undamping an exponential mixture of inverse Gaussian (EMIG) ccdf's. The EMIGs were discussed in Section 8 of [3].

Introducing a slight change of notation, we start with the Laplace transform of an EMIG pdf

$$
\hat{f}(s)=\frac{\mu+1}{\mu+\sqrt{1+s}} .
$$

Formula (3.1) is obtained from (8.9) of [3] by first replacing $\mu$ by $\mu+1$ and then introducing the scale parameter $\omega \equiv 1 / 2(\mu+1)$; i.e., $\hat{f}(s)=\hat{\rho}(s ; \omega, \mu+1) \equiv \hat{\rho}(\omega s, 1, \mu+1)$ for that $\omega$. Paralleling $\hat{g}(s)$ in (2.1), an extra scale parameter can be added to $\hat{f}(s)$ in (3.1).

The moments of the pdf with transform in (3.1) can be derived from the inverse Gaussian moments by using (8.3) and (8.10) of [3] ( $r$ should be $n$ in (8.3)). They are

$$
m_{1}(F)=\frac{1}{2(\mu+1)}, \quad m_{n+1}(F)=\frac{1}{(2+2 \mu)^{n+1}} \sum_{k=0}^{n} \frac{(n+1-k)(n+k) !}{k !}\left(\frac{\mu+1}{2}\right)^{k}
$$

and squared coefficient of variation (variance divided by the mean) $c^{2}=\mu+2$. For the case $\mu=1,(3.1)$ is the BME transform $\hat{v}(1 / 2,3 / 2 ; s)$ studied in [4] and the moments in this case are $m_{n}=n ! \beta_{n} /(n+1)$ where $\beta_{n}=\left(\begin{array}{c}2 n \\ n\end{array}\right) 4^{-n}$.

Paralleling (8.13) and (8.14) of [3], the ccdf has the Laplace transform

$$
\begin{aligned}
\hat{F}^{c}(s) & =\frac{1-\hat{f}(s)}{s}=\frac{1}{(\mu+\sqrt{1+s})(1+\sqrt{1+s})} \\
& =\frac{1}{\mu-1}\left(\frac{1}{1+\sqrt{1+s}}-\frac{1}{\mu+\sqrt{1+s}}\right), \quad \mu \neq 1 .
\end{aligned}
$$

From (3.4) we see that EMIG stationary-excess pdf is

$$
f_{e}(t)=\frac{\mu+1}{\mu-1} v(1 / 2,3 / 2 ; t)-\frac{2}{\mu-1} f(t)
$$

from which we obtain the simple moment recurrence for $\mu \neq 1$

$$
m_{n+1}(F)=\frac{n ! \beta_{n}}{2(\mu-1)}-\frac{n+1}{\mu^{2}-1} m_{n}(F) .
$$


The recurrence formula (3.6) is recommended over (3.2) to calculate the moments. It is noteworthy that the moments $m_{n}(F)$ are always integer sequences when $\mu$ is an integer and they are scaled by the factor $(2+2 \mu)^{n}$. Except for the cases $\mu=0$ and 1 , none of these integer sequences are found in Sloane and Plouffe [12]. For example, the moment sequence for $\mu=2$ is $1,5,51,807,17445,479565, \ldots$

From (3.1) and 29.3.37 of Abramowitz and Stegun [6],

$$
f(t)=(\mu+1)\left(\frac{e^{-t}}{\sqrt{\pi t}}-\mu e^{\left(\mu^{2}-1\right) t} \operatorname{erfc}(\mu \sqrt{t})\right), \quad t \geq 0
$$

Going from (3.7) to (3.2) is surprisingly difficult. It can be done by applying the GosperZeilberger algorithm, e.g., see Section 5.8, especially p. 236, of Graham, Knuth and Patashnik [10] or Petkovsek, Wilf and Zeilberger [11]. The associated EMIG pdf in [3], which unfortunately was inadvertently omitted from (8.10) of [3], is

$$
\rho(t ; 1, \nu)=\frac{\nu e^{-t / 2 \nu}}{\sqrt{2 \pi \nu t}}-2^{-1}(\nu-1) e^{(\nu-2) t / 2} \operatorname{erfc}((\nu-1) \sqrt{t / 2 \nu}) .
$$

To obtain (3.7) and (3.8), first scale $t$ by the factor $2 v$, then let $\nu=\mu+1$.

Similarly, from (3.4), we have for $\mu \neq 1$,

$$
F^{c}(t)=\frac{1}{\mu-1}\left(\mu e^{\left(\mu^{2}-1\right) t} \operatorname{erfc}(\mu \sqrt{t})-\operatorname{erfc}(\sqrt{t})\right), \quad t \geq 0
$$

whereas for $\mu=1$, we invert $(1+\sqrt{1+s})^{-2}$ to get

$$
F^{c}(t)=(1+2 t) \operatorname{erfc}(\sqrt{t})-2 \sqrt{\pi / t} e^{-t}, \quad t \geq 0 .
$$

In the case $\mu=1$, the pdf $f(t)$ in (3.7) coincides with the beta mixture of exponentials (BME) $\operatorname{pdf} v(1 / 2,3 / 2 ; t)$ in Abate and Whitt [4], which in turn coincides with the RBM first-moment pdf $h_{1}(t)$; see Table 3 in [4]. The associated cdf in $(3.10)$ is $v(3 / 2,3 / 2 ; t) / 4$. (See the next section for further discussion.)

For all $\mu>0$, the asymptotic expansion for $F^{c}(t)$ is

$$
F^{c}(t) \sim \frac{e^{-t}}{\sqrt{\pi t}} \sum_{n=1}^{\infty}(-1)^{n+1} k_{n}(\mu) n ! \beta_{n} t^{-n} \quad \text { as } \quad t \rightarrow \infty
$$

where $\beta_{n}=\left(\begin{array}{c}2 n \\ n\end{array}\right) 4^{-n}$ is the moment sequence of the gamma pdf $\gamma(t)=e^{-t} / \sqrt{\pi t}$ as in Table 3 of $[4]$ and

$$
k_{n}(\mu)=\sum_{k=0}^{2 n-1} \mu^{k}=\frac{1}{\mu-1}\left(1-\frac{1}{\mu^{2 n}}\right),
$$

drawing on 7.1.23 of Abramowitz and Stegun [6]. Note that $k_{n}(1)=2 n$. 
As in our construction of $\mathrm{B}_{2} \mathrm{ME}$ ccdf's from BME ccdf's in [4], we define the ccdf $G^{c}$ associated with $\hat{g}(s)$ in $(2.1)$ by undamping the $\operatorname{ccdf} F^{c}(t)$, i.e., by letting

$$
G^{c}(t)=e^{t} F^{c}(t), \quad t \geq 0
$$

Combining (3.3) and (3.13), we obtain

$$
\hat{G}^{c}(s)=\hat{F}^{c}(s-1)=\frac{1}{(\mu+\sqrt{s})(1+\sqrt{s})}
$$

and

$$
\hat{g}(s)=1-s \hat{G}^{c}(s)=1-\frac{s}{(\mu+\sqrt{s})(1+\sqrt{s})},
$$

just as in (2.1). Moreover,

$$
\hat{G}_{e}^{c}(s) \equiv \frac{1-\hat{g}_{e}(s)}{s}=\left(\frac{\mu+1}{\mu}\right) \frac{1}{\sqrt{s}(1+\sqrt{s})}+\left(\frac{1}{\mu(1-\mu)}\right) \frac{1}{1+\sqrt{s}}-\left(\frac{1}{\mu(1-\mu)}\right) \frac{1}{\mu+\sqrt{s}},
$$

so that, by 29.3.37 and 29.3.43 of Abramowitz and Stegun [6],

$$
G_{e}^{c}(t)=\frac{\mu}{1-\mu}\left(\mu^{-1} \psi\left(\mu^{2} t\right)-\psi(t)\right), \quad t \geq 0
$$

for $\psi$ in $(2.5)$.

In the case $\mu=1$, we can apply the BME and $\mathrm{B}_{2} \mathrm{ME}$ calculus in [4], in particular, (1.20), (1.7) and Table 3, to get

$$
\begin{aligned}
g_{e}(t)=G^{c}(t) & =V_{2}^{c}(1 / 2,3 / 2 ; t)=e^{t} V(1 / 2,3 / 2 ; t) \\
& =(1 / 4) e^{t} v(3 / 2,3 / 2 ; t) \\
& =(2 t+1) \psi(t)-2 \sqrt{t / \pi}
\end{aligned}
$$

and

$$
\begin{aligned}
G_{e}^{c}(t) & =V_{2}^{c}(3 / 2,1 / 2 ; t)=e^{t} V^{c}(3 / 2,1 / 2 ; t) \\
& =(3 / 4) e^{t} v(5 / 2,1 / 2 ; t) \\
& =2 \sqrt{t / \pi}-(2 t-1) \psi(t)
\end{aligned}
$$

as given in (2.8).

\section{Representation as a Mixture of Exponentials}

We now show that EMIGs and undamped EMIGs are both completely monotone; i.e., can be expressed as mixtures of exponentials. As a consequence, they can be approximated arbitrarily closely by hyperexponential (finite mixtures of exponential) distributions; see Feldmann 
and Whitt [9]. Of course, the hyperexponential approximations never match the asymptotic tail behavior. Nevertheless, the associated M/G/1 waiting-time distributions are also matched arbitrarily closely; see [9].

Theorem 4.1. An EMIG is completely monotone; in particular, the ccdf can be expressed as

$$
F^{c}(t)=\int_{0}^{1} e^{-t / y} w(y) d y
$$

where

$$
w(y)=\frac{\mu+1}{\pi \sqrt{y}}\left(\frac{\sqrt{1-y}}{1+\left(\mu^{2}-1\right) y}\right), \quad 0 \leq y \leq 1 .
$$

Proof. We regard the Laplace transform $\hat{F}^{c}(s)$ in (3.4) as the Stieltjes transform of the spectral density; i.e., initially assuming that

$$
F^{c}(t)=\int_{0}^{\infty} e^{-x t} \phi(x) d y
$$

we obtain

$$
\hat{F}^{c}(s)=\int_{0}^{\infty} \frac{1}{s+x} \phi(x) d x .
$$

We can then calculate the alleged spectral density $\phi(x)$ by inverting its Stieltjes transform, p. 126 of Widder [14]; i.e.,

$$
\phi(x)=-\frac{\operatorname{Im} \hat{F}^{c}(-x)}{\pi}=\frac{1}{\pi(\mu-1)}\left(\frac{\sqrt{x-1}}{x}-\frac{\sqrt{x-1}}{x+\mu^{2}-1}\right)=\frac{(\mu+1) \sqrt{x-1}}{\pi x\left(x+\mu^{2}-1\right)}, \quad x>1 .
$$

The mixing density $w(y)$ is related to the spectral density $\phi(x)$ by $w(y)=y^{-2} \phi\left(y^{-1}\right)$. Hence, from (4.5) we obtain (4.2).

We can combine (3.13) and Theorem 4.1 to obtain a corresponding result for undamped EMIGS.

Corollary 1. An undamped EMIG is also completely monotone, i.e.,

$$
\begin{aligned}
G^{c}(t) & =\int_{0}^{1} e^{-t(1-y) / y} w(y) d y \\
& =\int_{0}^{\infty} e^{-t / z} w(z /(z+1))(1+z)^{-2} d z
\end{aligned}
$$

for $w(y)$ in (4.2).

In two special cases the EMIG is a beta mixture of exponentials (BME), as considered in [4]. Recall that the beta density is

$$
b(p, q ; y)=\frac{\Gamma(p+q)}{\Gamma(p) \Gamma(q)} y^{p-1}(1-y)^{q-1}, \quad 0 \leq y \leq 1
$$


Corollary 2. For $\mu=0, w(y)=b(1 / 2,1 / 2 ; y)$; for $\mu=1, w(y)=b(1 / 2,3 / 2 ; y)$.

Hence, in the notation of [4], the EMIG in $(3.1)$ is $\nu(1 / 2,1 / 2 ; t)$ when $\mu=0$ and $\nu(1 / 2,3 / 2 ; t)$ when $\mu=1$. For those cases additional properties are given in [4]. Recall that the special case considered by Boxma and Cohen [7] is $\mu=1$. Thus their case is the $\mathrm{B}_{2} \mathrm{ME}$ pdf $\nu_{2}(1 / 2,3 / 2 ; t)$. By Theorem 8 of [4], it can also be expressed as a gamma mixture of Pareto distributions.

More generally, we can express the mixing pdf $w(y)$ in (4.2) as a linear combination of beta pdf's. To do so, we expand $\left(1+\left(\mu^{2}-1\right) y\right)^{-1}$ in $(4.2)$ in a power series.

Theorem 4.2. For $\mu>0$ with $\mu \neq 1$,

$$
w(y)=\frac{\mu+1}{2} \sum_{n=0}^{\infty}\left(1-\mu^{2}\right)^{n} \frac{\beta_{n}}{n+1} b\left(\frac{2 n+1}{2}, 3 / 2 ; y\right) .
$$

where $\beta_{n} \equiv\left(\begin{array}{c}2 n \\ n\end{array}\right) 4^{-n}$, the moments of $b(1 / 2,1 / 2 ; y)$.

\section{Time Asymptotics}

Combining (3.9) and (3.13), we obtain the undamped EMIG ccdf $G^{c}(t)$. From that form, we can obtain the asymptotics as $t \rightarrow 0$ and as $t \rightarrow \infty$. In particular, from (3.11),

Theorem 5.1. For the undamped EMIG distribution,

$$
\begin{aligned}
G^{c}(t) & \sim 1-2(\mu+1) \sqrt{t / \pi} \quad \text { as } \quad t \rightarrow 0, \\
G^{c}(t) & \sim\left(\frac{\mu+1}{2 \mu^{2}}\right) \frac{1}{\sqrt{\pi t^{3}}} \quad \text { as } \quad t \rightarrow \infty,
\end{aligned}
$$

and

$$
G_{e}^{c}(t) \sim\left(\frac{\mu+1}{\mu}\right) \frac{1}{\sqrt{\pi t}} \quad \text { as } \quad t \rightarrow \infty
$$

Similarly, we obtain the large-time asymptotics for $W^{c}(t)$ from (2.13). For other M/G/1 waiting-time asymptotics, see Willekens and Teugels [15], Abate, Choudhury and Whitt [5] and Boxma and Cohen [7].

Theorem 5.2. with the undamped EMIG service-time pdf transform $\hat{g}(s)$ in (2.1),

$$
W^{c}(t) \sim \frac{\rho}{1-\rho} G_{e}^{c}(t)\left[1-\frac{(1+\mu)^{2}-2(1-\rho) \mu}{2(1-\rho)^{2} \mu^{2} t}\right] \quad \text { as } \quad t \rightarrow \infty .
$$

Formula (5.4) here agrees with formula (3.12) of Boxma and Cohen [7] for the case $\mu=1$. 


\section{Heavy-Traffic Asymptotics}

Boxma and Cohen [7] establish general heavy-traffic limits and approximations as $\rho \rightarrow 1$. We obtain their result for our special case directly from the explicit representation in Section 2.

Theorem 6.1. If $\rho \rightarrow 1$, then $\nu_{1} \rightarrow 1+\mu, \nu_{2} /(1-\rho) \rightarrow \mu /(1+\mu)$ and

$$
W^{c}(t / \alpha) \psi(t)
$$

for $\psi(t)$ in (2.5), where

$$
\alpha=\frac{(1-\rho)^{2}}{\rho^{2}}\left(\frac{\mu}{1+\mu}\right)^{2} .
$$

Based on (6.1), we would use the approximation

$$
W^{c}(t) \approx \psi(\alpha t)=e^{\alpha t} \operatorname{erfc}(\sqrt{\alpha t})
$$

for $\alpha$ in (6.2). Since $\rho^{2} \rightarrow 1$ as $\rho \rightarrow 1$, the factor $\rho^{2}$ in (6.2) plays no role in the heavytraffic limit. However, it makes the heavy-traffic approximation (6.3) asymptotically correct as $t \rightarrow \infty$ for each $\rho$ as well. We could further simplify the right side of (6.3) by replacing $\operatorname{erf} c(\sqrt{\alpha t})$ by its asymptotic form as $\alpha \rightarrow 0$, but the numerics performed by Boxma and Cohen [7] show that it is better to keep the error function. This phenomenon very closely parallels our asymptotic normal approximation for the $\mathrm{M} / \mathrm{G} / 1$ busy-period distribution in Abate and Whitt [2]. Indeed, the same approximating functions are involved.

\section{Other Explicit Expressions}

Smith [13] first observed that if the service-time distribution has rational Laplace transform, then so does the $\mathrm{M} / \mathrm{G} / 1$ steady-state waiting-time distribution, so that at least in principle it can be inverted analytically. This is easy to see in two steps: (1) going from the service-time cdf $G$ to its associated stationary-excess cdf $G_{e}$ and (2) going from $G_{e}$ to the waiting-time cdf exploiting the Pollaczek-Khintchine formula. The other explicit representations obtained so far can be viewed as generalizations of this result. If the service-time distribution has a Laplace transform that is a rational function of $s^{1 / n}$, then it is easy to see that so does the $\mathrm{M} / \mathrm{G} / 1$ steady-state waiting-time distribution. For general $n$, this property seems difficult to

exploit, but for $n=2$, we can exploit it, because we can relate the transform involving $\sqrt{s}$ to the error function. 
For example, at least in principle, we can obtain the explicit $M / G / 1$ waiting-time distribution when the service-time distribution is a mixture of $k$ undamped EMIGs. By the usual partial fraction expansion (assuming no multiple roots), we can represent the waiting-time distribution as a linear combination of undamped EMIGs. However, the additional complexity seems to make this approach unattractive. 


\section{References}

[1] J. Abate and W. Whitt, The Fourier-series method for inverting transforms of probability distributions, Queueing Systems 10 (1992), 5-88.

[2] J. Abate and W. Whitt, Limits and approximations for the busy-period distribution in single-server queues, Prob. Eng. Inf. Sci. 9 (1995), 581-602.

[3] J. Abate and W. Whitt, An operational calculus for probability distributions via Laplace transforms, Adv. Appl. Prob. 28 (1996), 75-113.

[4] J. Abate and W. Whitt, Beta mixtures of exponential distributions, 1997, submitted.

[5] J. Abate, G. L. Choudhury and W. Whitt, Waiting-time tail probabilities in queues with long-tail service-time distributions, Queueing Systems 16 (1994), 311-338.

[6] M. Abramowitz and I. A. Stegun, Handbook of Mathematical Functions, National Bureau of Standards, Washington, D.C., 1972.

[7] O. J. Boxma and J. W. Cohen, The M/G/1 queue with heavy-tailed service-time distribution. CWI, Amsterdam, 1997.

[8] J. W. Cohen, The Single Server Queue, second ed., North-Holland, Amsterdam, 1982.

[9] A. Feldmann and W. Whitt, Fitting mixtures of exponentials to long-tail distributions to analyze network performance models, Performance Evaluation 31 (1997), 245-279.

[10] R. L. Graham, D. E. Knuth and O. Patashnik, Concrete Mathematics, second ed., Addison-Wesley, Reading, MA, 1994.

[11] N. Petkovsek, H. Wilf and D. Zeilberger, $A=B$, Peters, Wellesley, MA, 1996.

[12] N. J. A. Sloane and S. Plouffe, Encyclopedia of Integer Sequences, Academic, New York, 1995.

[13] W. L. Smith, On the distribution of queueing times, Proc. Camb. Phil. Soc. 49 (1953), 449-461.

[14] D. V. Widder, An Introduction to Transform Theory, Academic Press, New York, 1971.

[15] J. E. Willekens and J. L. Teugels, Asymptotic expansions for waiting time probabilities in an M/G/1 queue with long-tailed service time, Queueing Systems 10 (1992), 295-312. 